\title{
Médulloblastome extra-axial dans l’angle ponto-cérébelleux : A propos d'un cas avec revue de la littérature
}

\author{
Extra-axial medulloblastoma in cerebello-pontine angle: case report and litterature \\ review
}

\author{
Souad Daoud', Abdelmoumene Ryad Ayoun², Elmahdi Mustapha Mimouni, Abbes Casmi4, Maamer \\ Bouchakour 5
}

1 maitre assistante en neurochirurgie; service de neurochirurgie CHU d'ORAN- Faculté de médecine d'Oran- Algérie 2 maitre assistant en neurochirurgie; service de neurochirurgie CHU d'ORAN-Faculté de médecine d'Oran -Algérie 3 maitre assistant en neurochirurgie; service de neurochirurgie CHU d'ORAN-Faculté de médecine d'Oran -Algérie 4 assistant en neurochirurgie; service de neurochirurgie CHU d'ORAN Algérie

5 professeur, chef de service de neurochirurgie CHU d'ORAN Faculté de médecine d'Oran -Algérie

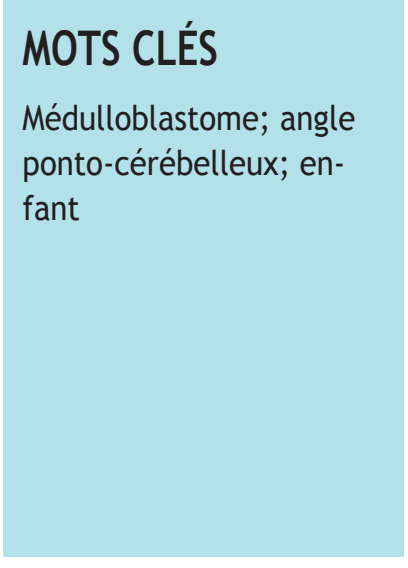

\section{KEY WORDS}

Medulloblastoma; cerebello-pontine angle; child.

\section{Résumé}

Nous rapportons un cas rare de médulloblastome extra axial siégeant au niveau de l'angle ponto-cérébelleux chez une enfant de 4 ans. Cette tumeur s'est manifestée par un tableau d'hyper tension intracrânienne avec altération de l'état de conscience évoluant depuis deux mois. Le scanner cérébral retrouvait une masse spontanément hyper dense plaquée contre la paroi postérieure du rocher gauche associée a une dilatation modérée du système ventriculaire sus-tentoriel. La patiente a été opérée en urgence de sa tumeur cérébrale. L'évolution clinique post opératoire était satisfaisante. Le diagnostic de médulloblastome a été confirmé par l'histopathologie.

\begin{abstract}
We report a rare case of an extra-axial medulloblastoma at the cerebello-pontine angle. This case concerns a 4-year-old child with an intracranial hypertension syndrome with alterations in the state of consciousness evolving for two months. Cerebral scanning found a mass spontaneously hyper dense plated against the posterior wall of the associated left rock with a moderate dilatation of the ventricular system. Patient was operated in emergency of her brain tumor. The postoperative clinical evolution was satisfactory; the cerebral CT of control confirmed the total excision of the tumor. The diagnosis of medulloblastoma was confirmed by histopathology.
\end{abstract}




\section{Introduction}

Le médulloblastome est une tumeur neuro-ectodermique primitive maligne (primitive neuro-ectodermic tumor PNET) [1], de grade IV histologique selon la classification de l'OMS [2]. Elle représente 20\% de toutes les tumeurs du système nerveux central (SNC) de l'enfant et 30\% des tumeurs de la fosse cérébrale postérieurs (FCP). Deux pics de fréquence ont été rapportés dans la littérature, le premier étant entre 03 et 04 ans et le second se situe entre 08 et 09 ans. Les garçons sont plus touchés que les filles avec un sex-ratio de 1.7. Les médulloblastomes se développent principalement aux dépens du vermis (partie médiane du cervelet) et du 4 ème ventricule.

Le site extra-axial de cette tumeur demeure une rareté $[3,4,6]$; nous en rapportons un cas.

\section{Observation}

Il s'agit d'une fille de 04 ans née d'une grossesse menée à terme, première d'une fratrie de deux enfants, qui a été admise au service de neurochirurgie du CHU d'Oran pour prise en charge d'un tableau d'hypertension intracrânienne, fait d'un état de somnolence avec des céphalées intenses soulagées par des vomissements en jet. La patiente trainait des céphalées minimes et quotidiennes depuis deux mois. L'examen neurologique initial était sans particularités, ne retrouvant notamment pas d'ataxie. Le développement staturo-pondéral était satisfaisant et aucun signe de déshydratation ou de malnutrition n’a été constaté. L'examen général a révélé des tâches « café au lait » au niveau du dos et de l'abdomen (plus de 15 tâches). Le scanner cérébral montrait une volumineuse formation tissulaire dense de l'angle ponto-cérébelleux gauche grossièrement ovalaire, mesurant $48 / 29 \mathrm{~mm}$, hétérogène par la présence de zones de nécrose, entourée d'œdème péri lésionnel, excentrée par rapport au conduit auditif interne gauche et compressive, responsable d'une hydrocéphalie tri-ventriculaire modérée en amont, faisant évoquer apriori un méningiome ou une neurofibromatose (maladie de Recklinghausen) (figure $1 \mathrm{a}$ et $1 \mathrm{~b}$ ) .

Figures 1 ( $a$ et b). TDM cérébrale objectivant une tumeur de l'angle ponto cérébelleux gauche.

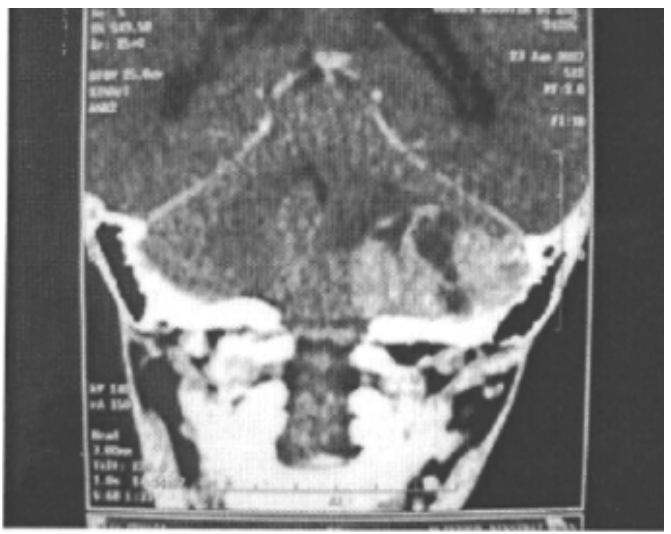

a. TDM cérébrale, coupe frontale

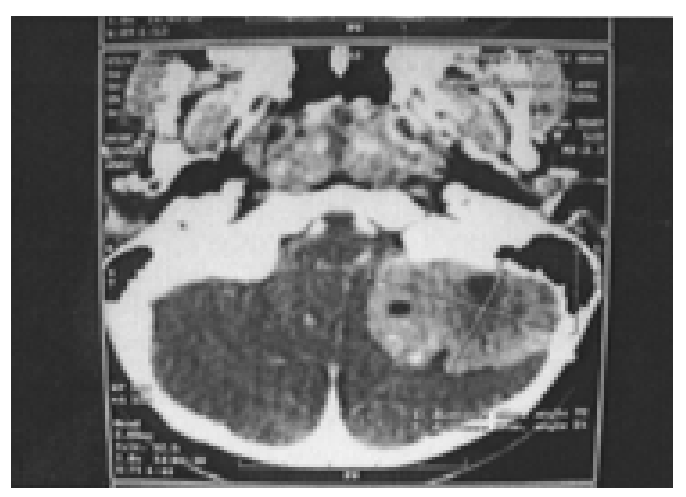

b. TDM cérébrale coupe axiale

Une craniectomie sous occipitale (abord de BUCY) a été réalisée en urgence et a mis en évidence une formation tumorale ovoïde blanc grisâtre, de consistance molle, avec présence de petits foyers nécrotiques. Nous avons procédé à une exérèse totale de cette tumeur.

Les suites post opératoires étaient favorables avec régression des céphalées et amélioration de l'état de conscience. La TDM cérébrale ainsi qu'une IRM médullaire de contrôle réalisées a un mois et a un an post-opératoire n'objectivaient aucun résidu tumoral au niveau de l'angle ponto-cérébelleux gauche (figures $2 \mathrm{a}, 2 \mathrm{2b}, 2 \mathrm{c}$ ).

Figures 1 ( $a$ et b). TDM cérébrale objectivant une tumeur de l'angle ponto cérébelleux gauche.

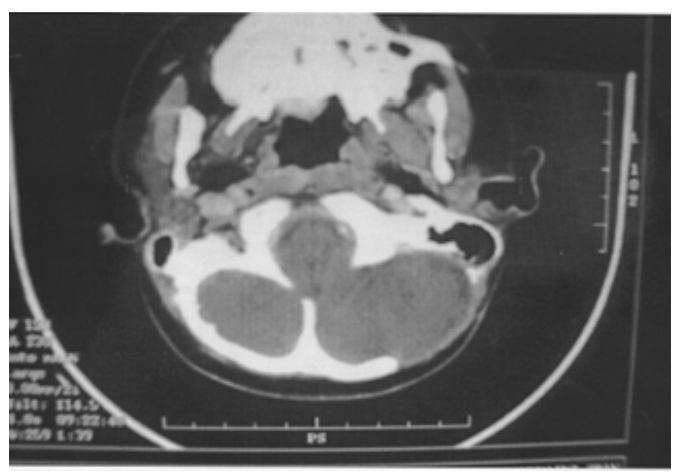

2a :Coupe axiale

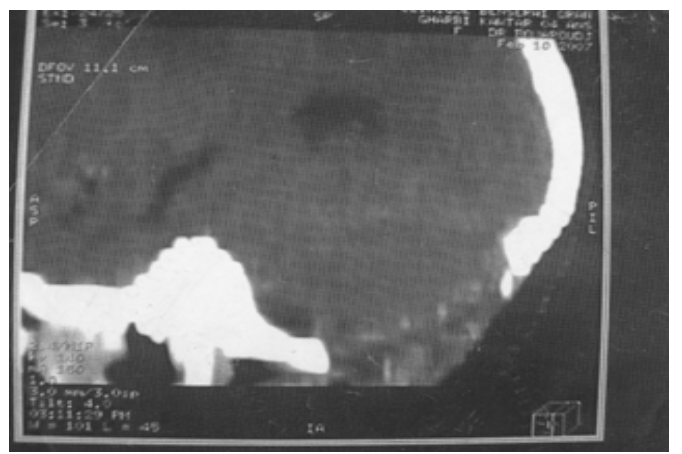

$2 \mathrm{~b}$ : coupe sagittale 


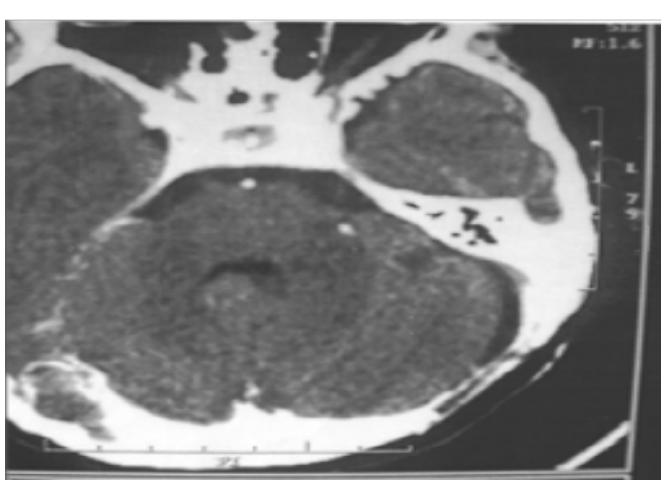

2c : TDM cérébrale de contrôle -coupe axiale un an après l'intervention

L'examen anatomopathologique de la pièce d'exérèse montrait une prolifération cellulaire très dense entourant les vaisseaux sanguins constituant des pseudo rosettes périvasculaires évoquant un médulloblastome (figure3).

Figure 3: aspect Histologie de la tumeur. prolifération cellulaire agencée en nappes denses et vastes formant des pseudo rosettes entourant les vaisseaux sanguins

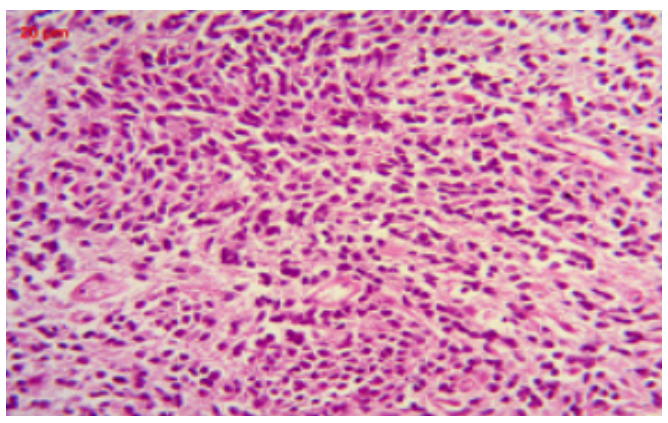

Vu l'âge de l'enfant (moins de 05 ans), le protocole médulloblastome $8 / 1$ n'est pas indiqué. De ce fait, une chimiothérapie a été préconisée selon le protocole BBSFOP.

L'évolution fut favorable et aucun signe d'extension de la tumeur n'a été observé lors du suivi IRM (cérébrale et moelle épinière) avec un recul de dix ans.

\section{Discussion}

Le médulloblastome est une tumeur neuroectodermique développée au niveau du vermis du cervelet $[6,7]$. Très rare chez l'adulte, c'est la plus fréquente des tumeurs cérébrales chez l'enfant. Environ $75 \%$ de ces tumeurs surviennent avant l'âge 15 ans, le pic de fréquence se situant autour de 5 ans. La tumeur se développe dans le toit du 4ème ventricule qu'elle finit par obstruer, provoquant une hydrocéphalie. Très rarement les médulloblastomes se développent dans l'angle ponto-cérébelleux (APC) ; 38 cas ont été rapportés dans la littérature jusqu' à ce jour dont 24\% (9/38) concernaient des enfants [7].

Une des particularités de cette tumeur est d'essaimer facilement le long du névraxe par voie méningée [8], et donc de provoquer des métastases à distance. A ce jour, les métastases rachidiennes du médulloblastome de l'angle ponto-cérébelleux n'ont pas été signalées [10].

La cause du médulloblastome n'est pas connue, mais une prédisposition génétique est observée chez environ $10 \%$ des patients, notamment dans le syndrome de Gorlin (gène Patched). D'autres syndromes génétiques peuvent être associés au médulloblastome; les plus fréquents sont la neurofibromatose de type 2 , le syndrome de Turcot ou encore le syndrome de Li Fraumeni.

Histologiquement, on distingue deux types : une forme classique qui est la plus fréquente, il s'agit d'une tumeur de couleur grisâtre friable nécrotique et hémorragique, constituée microscopiquement de petites cellules très denses regroupées en « rosettes » comme dans notre cas. La forme desmoplastique, beaucoup plus dense et ferme, est constituée de cellules se regroupant en lobules séparés de travées conjonctives, de topographie habituellement hémisphérique. La relation entre le type histologique du médulloblastome et sa localisation est encore incertaine et devrait être étudiée davantage, mais de nombreuses études ont suggéré que le type desmoplastique a été signalé plus fréquemment parmi les tumeurs extra axiales [5].

La présentation clinique du médulloblastome extra axial est variable, mais la plupart des patients présentent des antécédentes de céphalées évolutives durant moins de 3 mois dans $75 \%$ des cas. L'hydrocéphalie associée est responsable d'une proportion significative de la présentation clinique [8]. II n'existe aucun critère radiologique pathognomonique de la localisation extra axiale du médulloblastome.

Le traitement du médulloblastome de l'angle ponto-cérébelleux est le même que celui d'un médulloblastome vermien. Certains auteurs préconisent une dérivation systématique de l'hydrocéphalie soit par shunt ventriculo-péritonéal soit par voie endoscopique (ventriculo-cisternostomie) comme première étape ; puis l'excision tumorale dans un second temps. D'autres préconisent un drainage externe du liquide cérébro-spinal et une excision tumorale directe, comme dans notre cas, suivie d'une chimiothérapie et/ou d'une radiothérapie [4].

Concernant le pronostic, il n'existe actuellement aucun consensus clair permettant de savoir si les médulloblastomes extra-axiaux sont plus agressifs que leurs homologues de la ligne médiane [5].

\section{Conclusion}

Le médulloblastome est la tumeur pédiatrique maligne la plus 
fréquente de la fosse cérébrale postérieure ; elle se produit habituellement dans la ligne médiane. L'angle ponto-cérébelleux est une localisation très rare pour le médulloblastome en particulier chez l'enfant. La clinique et l'imagerie médicale ne peuvent pas aider à le distinguer des autres tumeurs de l'angle ponto cérébelleux. Bien que rare, le médulloblastome doit être pris en compte dans le diagnostic différentiel des lésions extra-axiales de la fosse postérieure. L'excision totale suivie d'une radiothérapie et / ou d'une chimiothérapie est nécessaire dans tous les cas de médulloblastome pour prévenir la récidive.

\section{Références bibliographiques}

[1] Kleihues P, Cavenee WK. Pathology and genetics: tumors of the nervous system. Lyon: IARC Press; 2000. World Health Organisation classification of tumors.

[2] Louis DN, Ohgaki H, Wiestler OD, Cavenee WK, Burger PC, Jouvet A, et al. The 2007 WHO classification of tumours of the central nervous system. Acta Neuropathol. 2007 Aug;114(2):97-109.

[3] Cugati G, Singh M, Ramamurthi R. Extra axial cerebellopontine angle medulloblastoma: A rare site of tumor. Indian J Med Paediatr Oncol.2011; 32(2):123-4.
[4] Fallah A, Bangalawala SM, Provias J, jha NK. Extra axial medulloblastoma in the Cerebellopontine angle. Can J surg. 2009; 52: E101-2.

[5] Bahrami E, Bakhti S, Fereshtehnejad SM, et al. Extra axial medulloblastoma in the cebellopontine angle: A report of a rare case with literaturereview . Med J Islam Repub Iran. 2014;13; 28:57.

[6] Chung EJ, Jeun SS. Extra axial medulloblastoma in the cebellarhemisphere. J KoreanNeurosurg Soc.2014;55(6):362-64.

[7] Louis DN, Ohgaki H, Wiestler OD, Cavenee WK, Burger PC, et al. (2007)

The 2007 WHO classification of tumours of the central nervous system. Acta Neuropathol 114: 97-109

[8] Kumar R, Achori G, Mishra A, et al. Medulloblastomas of the cebellopontine angle. Neurol India.2001;49:380-383.

[9] Mohan M, Pande A, Vasudevan MC, Ramamurthi R. Pediatricmedulloblastoma: A review of 67 cases at a single institute. Asian J Neurosurg.2008; 2:63-9.

[10] Stevenson L, Echlim F. Nature and origin of sometumors of cerebellum: Medullobastoma. ArchNeurolPsychiatry. 1934;31:93-109. 